\title{
Conformational dynamics of di-(perylene bisimide acrylate) and its footprints in steady-state, time-resolved, and fluorescence-correlation spectroscopy $\dagger$
}

\author{
Florian Spreitler, ${ }^{a}$ Michael Sommer, $\ddagger^{b}$ Mukundan Thelakkat ${ }^{b}$ and Jürgen Köhler ${ }^{\star a}$ \\ Received 8th February 2012, Accepted 12th April 2012 \\ DOI: $10.1039 / \mathrm{c} 2 \mathrm{cp} 40387 \mathrm{k}$
}

Employing steady-state spectroscopy, time-resolved fluorescence spectroscopy, and fluorescence (cross-) correlation spectroscopy we investigated di-(perylene bisimide acrylate) and compared the results with those from monomeric perylene bisimide acrylate. For the dimeric structure two emitting species were found. By comparison with the spectroscopic results from monomeric perylene bisimide one species was assigned to perylene moieties in an isolated, unstacked conformation, whereas the other species is attributed to aggregated $\pi$-stacked perylene moieties. The transition dynamics between these conformations was uncovered by fluorescence (cross) correlation spectroscopy. A consistent description of the various correlation curves is derived for a dynamic model that considers an additional non-fluorescent dark state that is associated exclusively with the aggregated species.

\section{Introduction}

Harnessing solar energy for renewable energy conversion becomes a major challenge for the future. At present one of the conversion technologies that offer long-term, large-scale use is photovoltaics, i.e. a technology based on inorganic solar cells manufactured in a classical top-down approach. ${ }^{1-3}$ Its natural counterpart however, photosynthesis, is based on exactly the opposite strategy - bottom-up, i.e. starting from individual molecules and combining them to supramolecular structures by self-assembly. Inspired by the various natural light-harvesting systems in photosynthesis, multichromophoric assemblies based on organic molecular building blocks have attracted considerable attention during the last decade. Methods have been explored to build artificial antennas, which are able to guide electronic excitation energy to an acceptor site for charge separation. Among the systems that have been studied are donor-acceptor dyads, dendrimers, J-aggregates, and cyclic chromophore arrays. ${ }^{4-21}$

Another promising platform for large-scale, low-cost, selfassembling photovoltaics is provided by $\pi$-conjugated materials. However, the advantages on the fabrication side (e.g. printability, structural flexibility) are accompanied by a high degree of

${ }^{a}$ Experimental Physics $I V$ and BIMF, University of Bayreuth, D-95440 Bayreuth, Germany.E-mail: juergen.koehler@uni-bayreuth.de; Fax: ( + 49) 921-554002

${ }^{b}$ Applied Functional Polymers, Department of Macromolecular Chemistry I, University of Bayreuth, D-95440 Bayreuth, Germany

$\dagger$ Electronic supplementary information (ESI) available. See DOI: $10.1039 / \mathrm{c} 2 \mathrm{cp} 40387 \mathrm{k}$

$\ddagger$ Present address: Melville Laboratory for Polymer Synthesis, Department of Chemistry, University of Cambridge, Cambridge, CB2 1EW, UK. complexity with respect to material selection and the physics of these systems. In organic molecules, due to low dielectric constants and electron-phonon coupling, optical excitations usually lead to the formation of an exciton, which is often associated with a spatially localized, strongly bound electron-hole pair. Therefore it became common practice to adopt the language of classical semiconductor physics also for the description of energy and charge-transfer processes in the organic manifold. Although, within this concept, the electronic energies are misleadingly deduced from (one-electron) molecular orbitals - rather than correctly from multi-particle electronic states - this approach has been exploited successfully for the description of many phenomena in the field of organic semiconductors. ${ }^{22,23}$ Within this terminology perylene bisimide (PBI) derivatives (also called perylene diimide or perylene tetracarboxylic diimide derivatives ${ }^{24,25}$ represent an important class of light absorbing n-type semiconductor molecules, exhibiting a relatively high electron affinity among large-bandgap materials. ${ }^{26,27}$ Owing to their excellent photochemical and thermal stability, in combination with a large extinction over a wide wavelength range of the solar spectrum, PBI derivatives became one of the most useful classes of chromophores, and are frequently used as sensitizers for organic solar cells, as fluorescence-based sensors or as markers in biomedical applications. ${ }^{26,28,29,30-36}$ However, the characteristic face-to-face $\pi-\pi$ stacking of PBI moieties leads to aggregation/crystallization, which drastically alters the efficiency of charge and energy transfer processes. ${ }^{37-43}$

Here we study dimers of perylene bisimide acrylate $\left((\mathrm{PerAcr})_{2}\right)$, Fig. 1, by steady-state spectroscopy, time-resolved spectroscopy, and fluorescence cross-correlation spectroscopy and compare 
the results with those from monomeric perylene bisimide acrylate (PerAcr) to shed light on the dynamics of the aggregation process and the concomitant change of the photophysical properties.

\section{Experimental}

\section{Sample preparation}

The chemical structures of the monomer PerAcr and the dimer $(\text { PerAcr })_{2}$ are shown in Fig. 1. PerAcr was synthesized as described elsewhere. ${ }^{44}$ The dimer (PerAcr) $)_{2}$ was obtained by polymerizing PerAcr via nitroxide mediated polymerization in dichlorobenzene and separating the resulting oligomeric mixture. A low initiator to the PerAcr ratio $(1: 10)$ was used together with a relatively short reaction time of $6 \mathrm{~h}$. The resulting mixture of oligomers was precipitated into acetone, filtered, dried and purified using a soxhlet apparatus with acetone to remove any remaining monomer PerAcr. The residual solid (a mixture of dimer and higher oligomers) was separated on a preparative gel permeation chromatography (GPC) column at a flow rate of $5 \mathrm{ml} \mathrm{min}{ }^{-1}$ in THF. Narrow fractions were taken, all of which contained mixtures of oligomers with a strong enrichment of particular oligomers. Between the second last and the last fraction, baseline resolution was observed on the preparative GPC. The individual fractions were then measured on an analytical GPC setup with THF as the eluent,

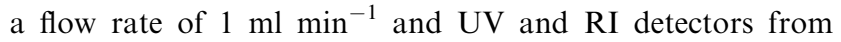
Waters. Polystyrene standards were used for calibration and 1,2-dichlorobenzene as an internal standard. The last fraction (containing the lowest molecular weight oligomer; $M_{\mathrm{n}}: 1600 \mathrm{~g} \mathrm{~mol}^{-1}$ ) had a very low polydispersity index (PDI) of 1.01 comparable to monodisperse compounds and did not show any shoulders on either side. Since the monomer PerAcr was already removed, we concluded that the last fraction contained the dimer only. The fractions were further characterized by MALDI-ToF, which confirmed the successful isolation of the dimer (see ESI $\dagger$ for GPC traces of oligomers and MALDI-ToF spectra).
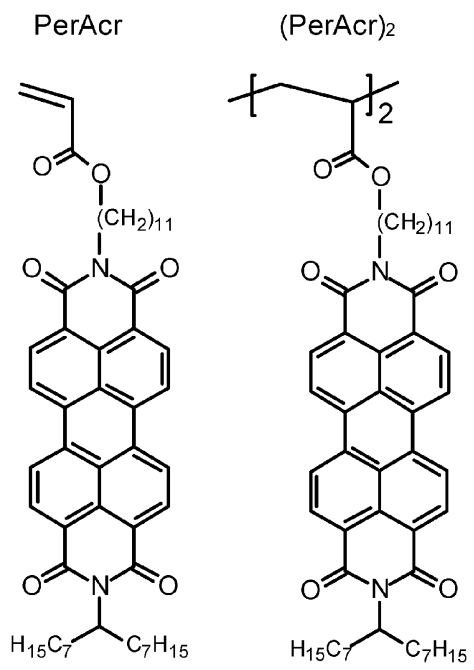

Fig. 1 Chemical structures of monomeric and dimeric perylene bisimide acrylates PerAcr and (PerAcr $)_{2}$, respectively.
The optical experiments were performed on solutions of PerAcr as well as (PerAcr $)_{2}$ in toluene at concentrations of $1 \mu \mathrm{M}$, except for the correlation spectroscopy where the concentration was lowered to $10 \mathrm{nM}$.

\section{Optical setup}

For the steady-state spectroscopy, the sample solutions were analyzed with commercial spectrometers (absorption: Perkin Elmer Lambda 9 UV/VIS/NIR spectrometer; fluorescence and fluorescence excitation: Varian Cary Eclipse fluorescence spectrophotometer). For the time-resolved fluorescence spectroscopy, the samples were excited at $479 \mathrm{~nm}$ with the frequency doubled output of a titanium:sapphire laser system (Tsunami, Newport Spectra-Physics) which provided pulses of 2 ps duration. Frequency doubling as well as controlling the repetition rate of the laser between $200 \mathrm{kHz}$ and $2 \mathrm{MHz}$ was accomplished with a pulse picker (Model 3980, Newport Spectra-Physics). The excitation energy per pulse was adjusted not to exceed $10 \mathrm{pJ}$. The polarisation of the excitation light was adjusted to the magic angle (with respect to the vertical direction) with a half-wave plate. Two achromatic lenses were used to focus the excitation onto the sample and to collect the emission under right angle conditions. In order to fulfil magic angle conditions for the detection, only the vertically polarized component of the signal was directed toward a spectrograph (250 Imaging Spectrograph, Bruker, spectral resolution $3 \mathrm{~nm}$ ). The signal was detected with a streak camera system (C5680 series, Hamamatsu) which was operated in single sweep mode and provided a temporal resolution of down to $15 \mathrm{ps}$, depending on the total time range of the experiment.

The fluorescence auto- and crosscorrelation experiments were carried out on a home built confocal microscope. The output of a continuous diode laser (Cyan 488-40, Newport Spectra-Physics), operating at $488 \mathrm{~nm}$, was reflected by a dichroic mirror (z488rdc, AHF) and focussed onto the sample by an oil-immersion objective $(60 \times$, N.A. 1.45 , Olympus $)$ that was also used to collect the emission. The excitation power was $100 \mu \mathrm{W}$ for all experiments which corresponds to an excitation intensity in the focal spot of $70 \mathrm{~kW} \mathrm{~cm}{ }^{-2}$. Spectral selectivity in the detection path was achieved by using dielectric filters (510 nm-550 nm; Brightline HC 531/40, AHF; $660 \mathrm{~nm}$ and above; EdgeBasic $635 \mathrm{LP}, \mathrm{AHF})$. For the autocorrelation measurements, the fluorescence first passed either the green or the red dielectric filter and was then split into two components by a $50: 50$ beam-splitter. For the crosscorrelation measurements, the fluorescence was split by a dichroic mirror (620 DCXR, AHF) and the reflected (transmitted) light passed the green (red) dielectric filter. For both types of experiments, the two components of the signal were focussed onto two lowbackground avalanche photodiodes (PDM $50 \mathrm{ct}$, PicoQuant), which also served directly as detection pinholes for the confocal microscope. The registered photons were processed by an ADWin Gold I/O device with 10 ns timing resolution (Jäger Computergesteuerte Messtechnik) and stored in computer memory. From these data, the crosscorrelation functions between the two signals were calculated by home written software. In fact, for the configuration using the $50: 50$ beam-splitter, these crosscorrelations correspond to autocorrelations, with the added 
value that detector specific artefacts like dead times and afterpulsing are surpassed resulting in a better time resolution of the experiment. ${ }^{45}$

All data were analysed using the freely distributed software gnuplot (version 4.2.3).

\section{Results}

\section{Steady-state spectroscopy}

The absorption spectra of PerAcr and (PerAcr) $)_{2}$ are shown in Fig. 2a. For PerAcr the spectrum features the characteristic fingerprint bands of the perylene bisimide (PBI) unit with an absorption maximum at $527 \mathrm{~nm}$ that is accompanied by a vibronic progression peaking at $490 \mathrm{~nm}$ and $460 \mathrm{~nm}$. The relative peak intensities of the bands are $6: 3: 1$. For $(\text { PerAcr })_{2}$ the absorption spectrum features bands at nearly the same spectral positions ( $528 \mathrm{~nm}, 492 \mathrm{~nm}$, and $463 \mathrm{~nm}$ ) as observed for PerAcr, yet with considerably different relative peak intensities $(2: 2: 1)$ and with an increased spectral bandwidth. The profiles of these spectra were independent of the concentration between $20 \mu \mathrm{M}$ and $0.2 \mu \mathrm{M}$.

The emission spectra of the two compounds are compared in Fig. $2 b$ for an excitation wavelength of $488 \mathrm{~nm}$. Three intensity maxima can be distinguished which occur at similar spectral positions for the two species, i.e. at $537 \mathrm{~nm}, 579 \mathrm{~nm}$, and $627 \mathrm{~nm}$ for PerAcr and at $537 \mathrm{~nm}, 580 \mathrm{~nm}$, and $626 \mathrm{~nm}$ for $(\mathrm{PerAcr})_{2}$. However, $(\mathrm{PerAcr})_{2}$ features a broad emission in the spectral range from $650 \mathrm{~nm}$ to $800 \mathrm{~nm}$ which is not observed for PerAcr. Moreover, the absolute peak emission intensity of the band at $537 \mathrm{~nm}$ (corrected for the difference in absorbance of the two samples at $488 \mathrm{~nm}$ ) was three times lower for (PerAcr) $)_{2}$ with respect to PerAcr. The profiles of these spectra were independent of the concentration between $10 \mathrm{nM}$ and $1 \mu \mathrm{M}$.

Additionally we performed fluorescence-excitation spectroscopy of $(\mathrm{PerAcr})_{2}$ as a function of the detection wavelength. For that purpose, the registration of the signal was restricted to a $20 \mathrm{~nm}$ wide spectral interval centred at a detection wavelength $\lambda_{\text {det }}$, which was varied in increments of $20 \mathrm{~nm}$ from $530 \mathrm{~nm}$ to $790 \mathrm{~nm}$. The respective spectra are shown in Fig. 2c together with a fluorescence-excitation spectrum from PerAcr (detected at $530 \mathrm{~nm}$ ) for comparison. Obviously, the spectral profile of the (PerAcr $)_{2}$ excitation spectrum shows strong variations as a function of the detection wavelength. For detection around $530 \mathrm{~nm}$ it resembles the excitation spectrum of PerAcr. Increasing the detection wavelength from $550 \mathrm{~nm}$ to $650 \mathrm{~nm}$ reveals a gradual change of the spectral profile towards a spectrum with maxima at $532 \mathrm{~nm}$ and $494 \mathrm{~nm}$ and relative peak intensities of $2: 3$, which remains nearly unaltered for detection at longer wavelengths. Moreover, at $511 \mathrm{~nm}$ and $538 \mathrm{~nm}$ the (PerAcr $)_{2}$ excitation spectra feature isosbestic points.

\section{Time-resolved fluorescence spectroscopy}

Fig. 3a shows an example of the experimental results obtained with the streak camera for (PerAcr) $)_{2}$ upon excitation at $479 \mathrm{~nm}$. In the horizontal direction the streak image covers the wavelength range from $490 \mathrm{~nm}$ to $655 \mathrm{~nm}$, and in the vertical
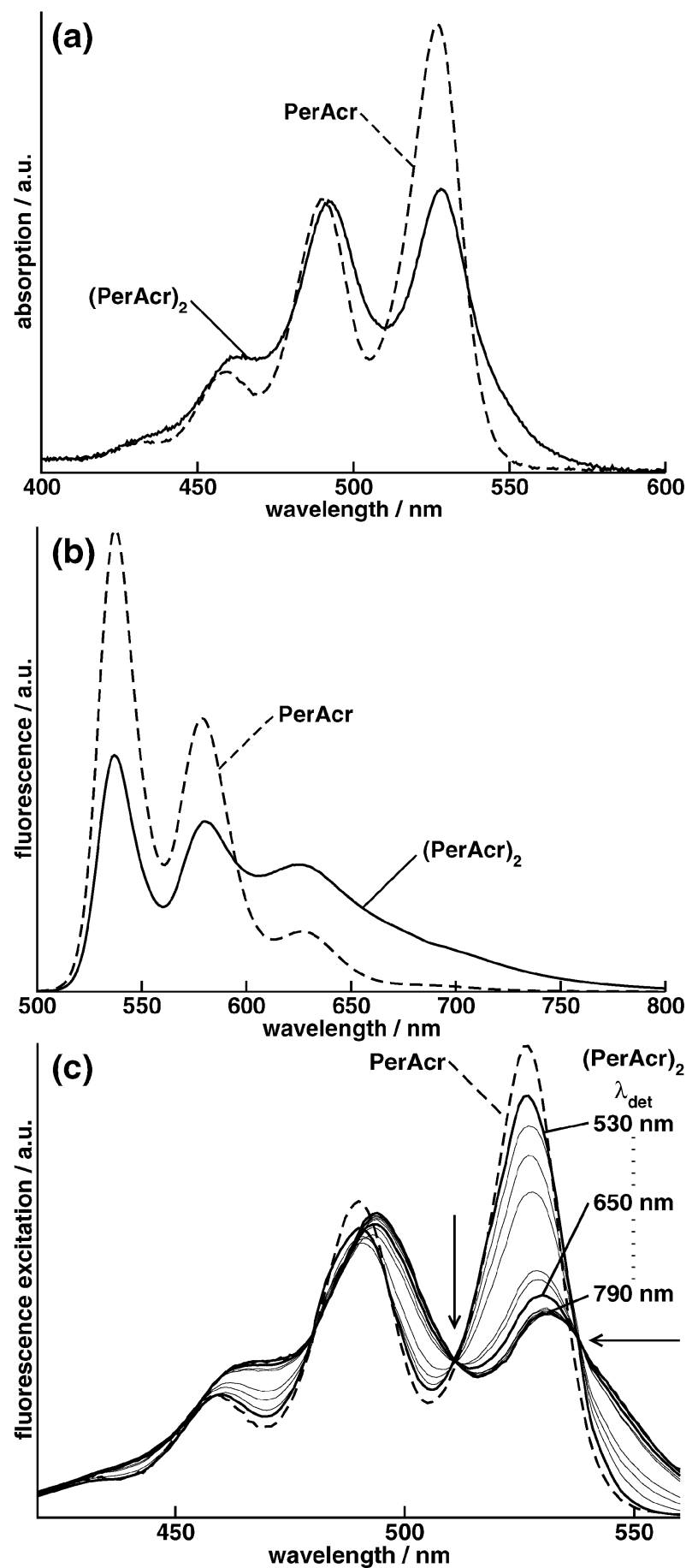

Fig. 2 Steady-state spectra of PerAcr (dashed lines) and (PerAcr) (solid lines) dissolved at $1 \mu \mathrm{M}$ concentration in toluene. For better comparison all spectra are normalised to the area. (a) Absorption spectra. (b) Emission spectra for excitation at $488 \mathrm{~nm}$. (c) Fluorescence-excitation spectra. For (PerAcr $)_{2}$ the spectra were registered selectively within a $20 \mathrm{~nm}$ wide interval centred around a wavelength $\lambda_{\operatorname{det}}$. The bold lines correspond to fluorescence-excitation spectra recorded for centre wavelengths $530 \mathrm{~nm}, 650 \mathrm{~nm}$, and $790 \mathrm{~nm}$, respectively. Isosbestic points at $511 \mathrm{~nm}$ and $538 \mathrm{~nm}$ are indicated by the arrows.

direction the streak image covers the time interval until $40 \mathrm{~ns}$ after the excitation pulse. The detected emission intensity is given by the grey scale, which is scaled by factors of $1,10,25$ and 50 

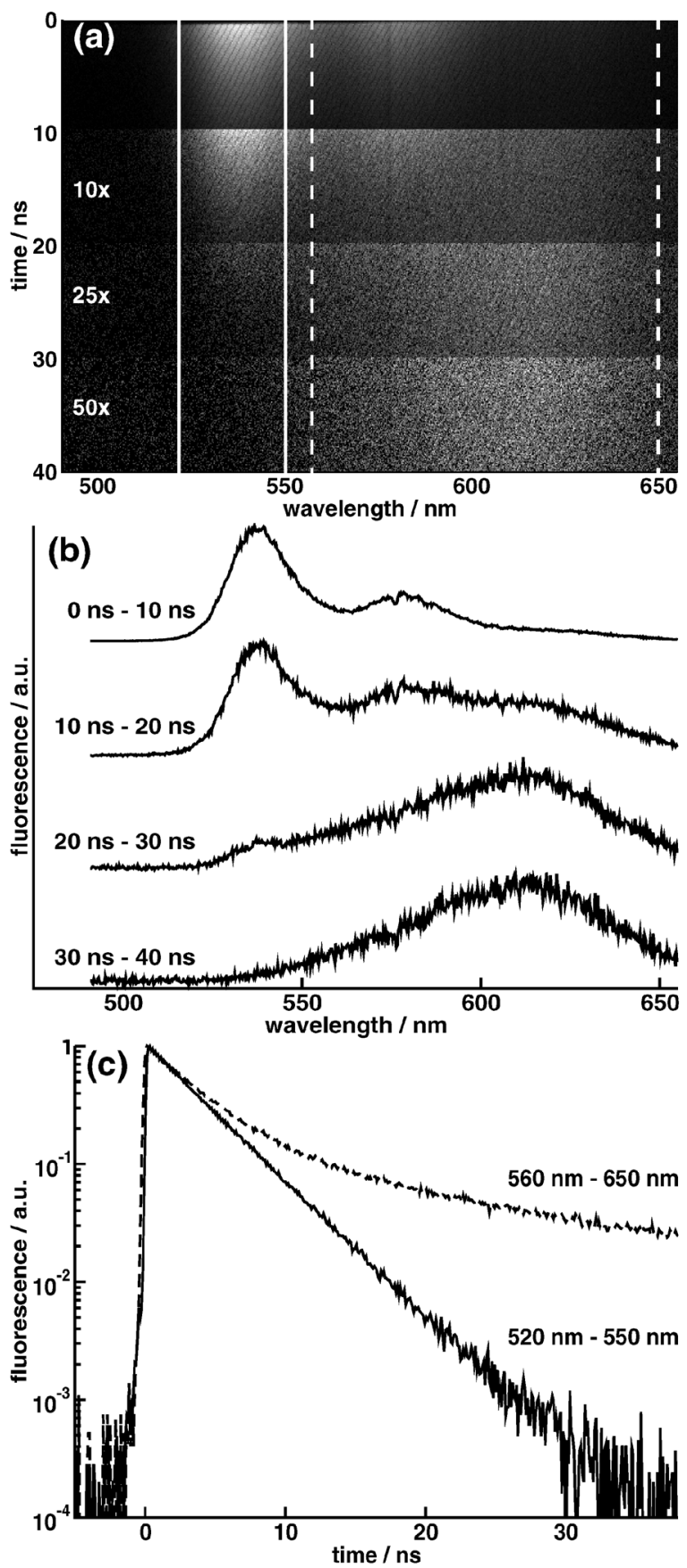

Fig. 3 Time-resolved emission spectroscopy of (PerAcr $)_{2}(1 \mu \mathrm{M}$ in toluene) excited at $479 \mathrm{~nm}$. (a) Streak image. The horizontal axis corresponds to the emission wavelength, the vertical axis corresponds to the time after the excitation pulse, and the emission intensity is given by a grey scale, where black represents low intensity and white represents high intensity. For better visibility, the emission intensity is scaled by factors of $1,10,25$ and 50 for consecutive intervals of $10 \mathrm{~ns}$ duration. The solid lines $(520 \mathrm{~nm}-550 \mathrm{~nm})$ and dashed lines $(560 \mathrm{~nm}-600 \mathrm{~nm})$ indicate spectral regions that are analysed separately in part (c). (b) Emission spectra that result from integration of the streak image along the time axis within the consecutive 10 ns intervals. The spectra are normalised to their peak values and offset from each other for clarity. (c) Fluorescence decays of the emission that results from integration of the streak image along the wavelength axis within the two spectral intervals marked in part (a). Both transients are normalised to their maximum. for consecutive intervals of $10 \mathrm{~ns}$ duration for better contrast. Integration of the streak image along the time axis over these intervals yields the time-resolved emission spectra shown in Fig. 3b. In the first 10 ns after excitation, the spectrum features maxima at $538 \mathrm{~nm}$ and $579 \mathrm{~nm}$ and negligible emission intensity beyond $600 \mathrm{~nm}$. In the interval $10 \mathrm{~ns}-20 \mathrm{~ns}$ after excitation, an additional shoulder at about $620 \mathrm{~nm}$ can be observed. During the last two time intervals the two initial peaks vanish (in the 20-30 ns interval a small remainder of the peak at $538 \mathrm{~nm}$ is still observable) while the shoulder in the red spectral range grows to a broad band peaking at $620 \mathrm{~nm}$. Along the wavelength axis the streak image has been divided into intervals from $520 \mathrm{~nm}-550 \mathrm{~nm}$ and from $560 \mathrm{~nm}-650 \mathrm{~nm}$, marked by the solid and dashed lines in Fig. 3a. Integration of the streak image along the wavelength axis within these intervals yields the decay kinetics of the (PerAcr $)_{2}$ emission in these spectral regions, as shown in Fig. 3c. The emission in the short-wavelength range decays mono-exponentially with a decay time of $3.7 \mathrm{~ns}$, whereas the decay of the emission in the long-wavelength range is compatible with a bi-exponential function with decay times of $3.7 \mathrm{~ns}$ and $27 \mathrm{~ns}$. In order to better resolve these components, we recorded streak images either in the spectral range from $490 \mathrm{~nm}-650 \mathrm{~nm}$ or in the spectral range from $630 \mathrm{~nm}-790 \mathrm{~nm}$, and for each spectral range in the time intervals (temporal resolution) $5 \mathrm{~ns}(30 \mathrm{ps})$, $50 \mathrm{~ns}(300 \mathrm{ps})$, and $500 \mathrm{~ns}$ ( $3 \mathrm{~ns})$ after the excitation pulse. The general observation was that although both decay times could be found in all streak images, the fast decay component (3.7 ns) was dominating at shorter wavelengths $(500 \mathrm{~nm}-660 \mathrm{~nm})$ whereas the slow decay component ( $27 \mathrm{~ns}$ ) was dominating at longer wavelengths (660 $\mathrm{nm}$ and above). A similar sequence of experiments was performed on PerAcr for comparison. This yielded exclusively mono-exponential decays with a time constant of 4 ns (data not shown).

\section{Fluorescence-correlation spectroscopy}

The results of fluorescence correlation experiments on (PerAcr $)_{2}$ are shown in Fig. 4: for the emission in the green spectral region $(510 \mathrm{~nm}-550 \mathrm{~nm})$ in Fig. 4a, for the emission in the red spectral region (beyond $660 \mathrm{~nm}$ ) in Fig. 4b, and for the cross correlation of the emission in the two spectral regions in Fig. 4c. The experimental data are overlaid with the results from global least-squares fits that were based on different models and will be discussed later.

For lag times longer than microseconds all observed correlations decay from a plateau value to zero on a time scale of some ten microseconds reflecting the diffusion of the emitters through the confocal volume. For the correlation of the green (red, cross) emission the respective data are $0.12(0.11,0.19)$ for the plateau value and $60 \mu \mathrm{s}(60 \mu \mathrm{s}, 50 \mu \mathrm{s})$ for the time it takes to decay to half of this value.

On the sub-microsecond time scale the three correlation curves feature significant differences. The autocorrelation of the green emission shows a pronounced peak with a half width at half maximum (HWHM) of $60 \mathrm{~ns}$, the amplitude of which exceeds the plateau value by a factor of 3 . At the coincidence point, i.e. at lag time zero, it exhibits a dip where the signal drops to about $80 \%$ of the maximum, see inset Fig. $4 a$. 

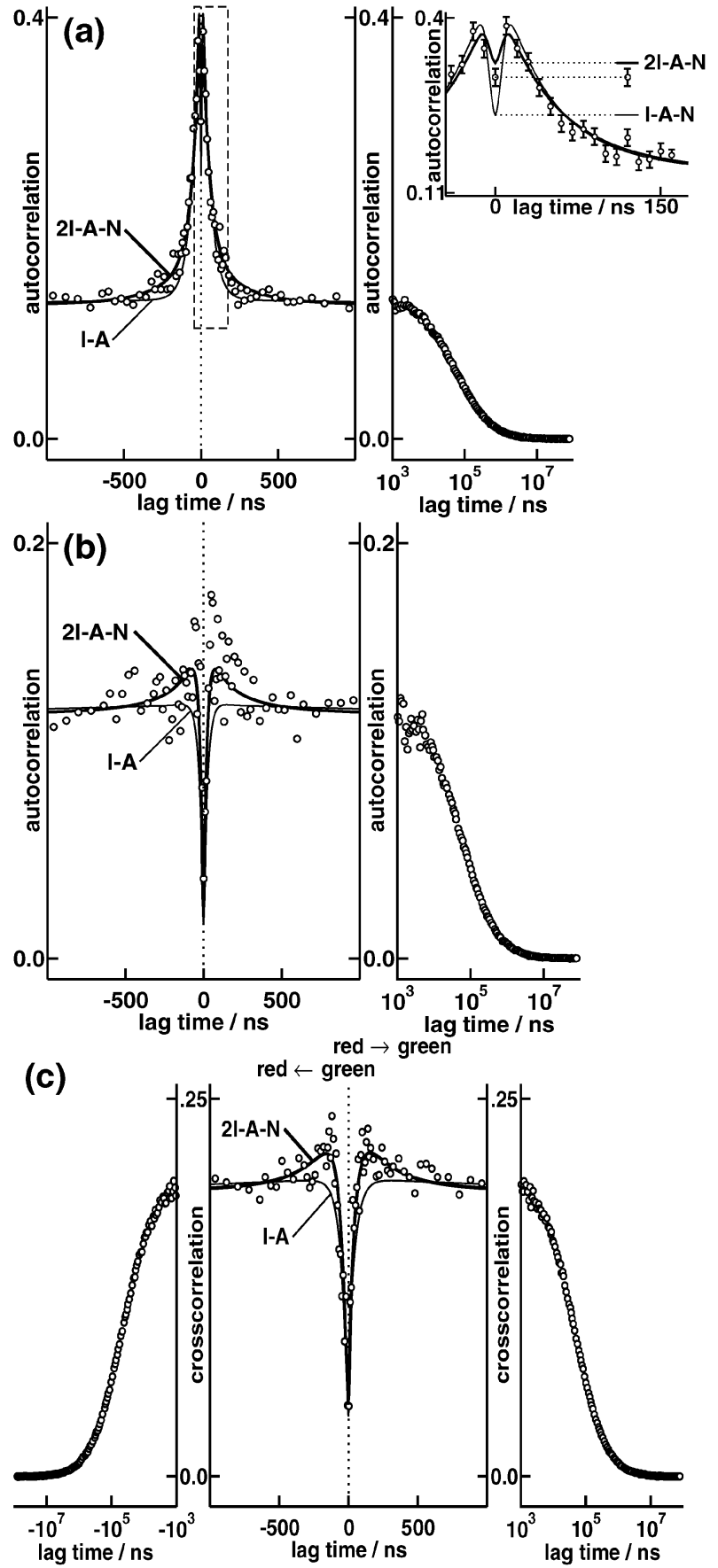

Fig. 4 Comparison of experimental correlation functions (open circles) obtained from a $10 \mathrm{nM}$ solution of $(\mathrm{PerAcr})_{2}$ in toluene, with calculated correlation functions based on the models discussed in the text (I-A model: thin line; 2I-A-N model: bold line). For all correlations the abscissa is plotted on a linear time scale for lag times $\pm 1 \mu \mathrm{s}$, and on a logarithmic time scale outside this interval. The coincidence points at lag time zero are marked by the vertical dotted lines. (a) Autocorrelation for the (PerAcr $)_{2}$ emission in the green spectral range $(510 \mathrm{~nm}-550 \mathrm{~nm})$. The inset corresponds to the dashed box of the main figure and compares the data with the calculated autocorrelations according to models $\mathrm{I}-\mathrm{A}-\mathrm{N}$ (thin line) and $2 \mathrm{I}-\mathrm{A}-\mathrm{N}$ (bold line) on an expanded scale. (b) Autocorrelation for the (PerAcr) $)_{2}$ emission in the red spectral range (660 $\mathrm{nm}$ and above). (c) Crosscorrelation between the red and green emission of (PerAcr) $)_{2}$. Positive (negative) lag time corresponds to green (red) emission that was detected after red (green) emission.
This dip consists of only one data point reflecting that its width is below the experimental resolution of $10 \mathrm{~ns}$. For the autocorrelation of the red emission the peak has a HWHM of $200 \mathrm{~ns}$ and exceeds the plateau value by a factor of 1.3 . At the coincidence point the intensity drops to $20 \%$ of the maximum and the HWHM of the dip is 20 ns. Finally, for the cross correlation the sub-microsecond peak has a HWHM of $300 \mathrm{~ns}$ and exceeds the plateau by a factor of 1.2. Interestingly the dip at the coincidence point, where the intensity drops to $20 \%$ of the maximum, has an asymmetric profile. Its HWHMs are $20 \mathrm{~ns}$ for positive lag times, corresponding to a situation where first a red photon and then a green photon is detected, and 40 ns for negative lag times, corresponding to a reversed chronology of the detected photons.

\section{Data analysis}

\section{Steady-state spectroscopy}

The dependence of the profile of the fluorescence-excitation spectra, Fig. 2c, on the detection wavelength provides already a hint that the optical spectra correspond to a superposition of contributions from several fluorescent species. If we denote the (area-normalised) fluorescence-excitation spectrum of species $i$ by $E_{i}(\lambda)$, we can express the (area-normalised) fluorescenceexcitation spectrum detected at the wavelength $\lambda_{\text {det }}$ as $E^{\left(\lambda_{\text {det }}\right)}(\lambda)=\sum_{i} p_{i}^{\left(\lambda_{\text {det }}\right)} E_{i}(\lambda)$, where $p_{i}^{\left(\lambda_{\text {det }}\right)}$ is the relative weight of species $i$ at the respective detection wavelength and $\sum_{i} p_{i}^{\left(\lambda_{\operatorname{det}}\right)}=1$. At an isosbestic point $\lambda_{0}$ this reads as $E^{\left(\lambda_{\text {det }}\right)}\left(\lambda_{0}\right)=$ $\sum_{i} p_{i}^{\left(\lambda_{\mathrm{det}}\right)} E_{i}\left(\lambda_{0}\right)=$ const. for all detection wavelengths. From the observation of two isosbestic points in the fluorescenceexcitation spectra and from the conjecture that it is highly unlikely that this equation is fulfilled at the same time by more than two species, we conclude that the fluorescence-excitation spectra reflect the contributions from exactly two fluorescent species. It is important to note that - in contrast to absorption spectroscopy - the observation of isosbestic points in fluorescenceexcitation spectra is not necessarily equivalent to a linear stoichiometric relationship between the two fluorescing species, and that no information can be gained about non-fluorescing species.

Led by these considerations, we approximated the (PerAcr) $)_{2}$ absorption spectrum as the sum of two contributions for which we used the area-normalised fluorescence-excitation spectra detected at $530 \mathrm{~nm}$ and $790 \mathrm{~nm}$, respectively. We found good agreement with the measured absorption spectrum for equal fractions from each component, see Fig. 5.

\section{Time-resolved fluorescence spectroscopy}

The idea that the optical spectra correspond to the superposition of contributions from two species is further corroborated by the finding that the fluorescence transients uncover two components with distinctly different time constants. It is known that perylene dyes show a strong tendency for $\pi-\pi$ stacking and comparison with literature data ${ }^{38-41,46,47}$ shows that a strong red-shift of the emission, accompanied by an increased decay time is common for $\pi-\pi$ stacked aggregates of perylene derivatives. Therefore we assign the red-shifted, 


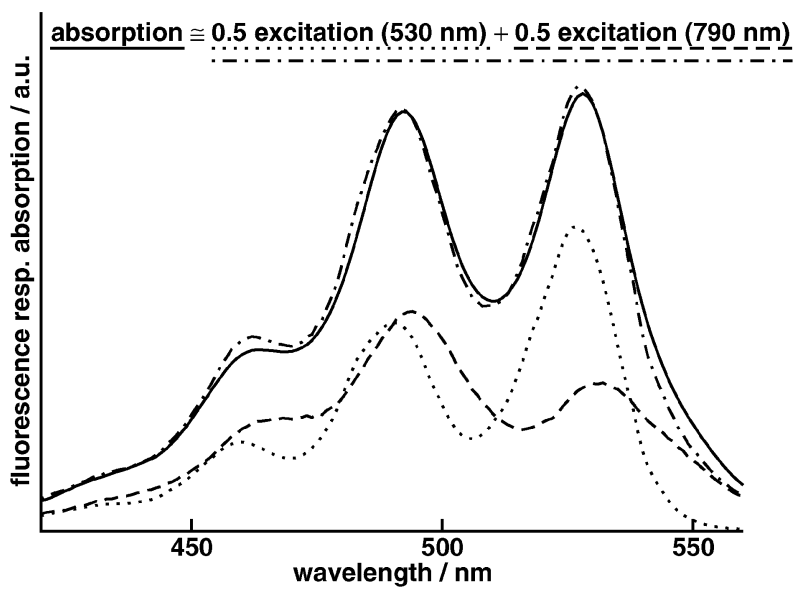

Fig. 5 Comparison of the (PerAcr) $)_{2}$ absorption spectrum (solid line) with the sum spectrum (dash-dotted line) of the (PerAcr) $)_{2}$ excitation spectra detected at $530 \mathrm{~nm}$ (dotted line) and $790 \mathrm{~nm}$ (dashed line), respectively. All spectra are normalised to the area; the two excitation spectra have been scaled by a factor of 0.5 , representing their relative contribution to the sum.

long-lived fluorescence component as well as the fluorescenceexcitation spectrum detected at $790 \mathrm{~nm}$ to originate from a stacked conformation of the (PerAcr $)_{2}$ molecules which will be referred to as AGG-(PerAcr $)_{2}$ in the following. The fast decay component of $3.7 \mathrm{~ns}$ in $(\text { PerAcr })_{2}$ is close to the $4.0 \mathrm{~ns}$ observed for PerAcr, and this decay component was found predominantly in the short wavelength range of the (PerAcr $)_{2}$ emission, for which the fluorescence-excitation spectrum resembles the one obtained for PerAcr. Thus, we assign this fluorescence component and the fluorescence-excitation spectrum detected at $530 \mathrm{~nm}$ to originate from an unstacked conformation of $(\text { PerAcr })_{2}$ referred to as ISO-(PerAcr $)_{2}$ in the following.

In order to substantiate these assignments we performed a global analysis of the recorded time-resolved (PerAcr) $)_{2}$ emission spectra. For this analysis each of the six streak images was divided on the wavelength axis into 31 intervals of $5 \mathrm{~nm}$ width each. The intervals were chosen such that an overlap between the two separately detected spectral regions was ensured. Integration of the streak image along the wavelength axis within each interval yielded 186 fluorescence decays, which were fitted using the following function:

$$
I^{(\lambda)}(t)=\left(A_{\mathrm{ISO}}^{(\lambda)} \mathrm{e}^{-\frac{t}{\tau_{\mathrm{ISO}}}}+A_{\mathrm{AGG}}^{(\lambda)} \mathrm{e}^{-\frac{t}{\tau_{\mathrm{AGG}}}}+A_{\text {fast }}^{(\lambda)} \delta(t)\right) \otimes \operatorname{IRF}(t)
$$

Here the superscript $\lambda$ refers to the centre wavelength of the respective $5 \mathrm{~nm}$ interval as defined above. In addition to the ISO-(PerAcr) $)_{2}$ and AGG-(PerAcr) $)_{2}$ components with amplitudes $A_{\text {ISO }}$ and $A_{\mathrm{AGG}}$, the term $A_{\text {fast }} \delta(t)$ was introduced to account for unresolved processes occurring on time scales faster than the time resolution of the experiment. $\otimes$ in eqn (1) denotes the convolution of the sum of these contributions with the Gaussian instrument response function (IRF) of the respective time range. In the fitting process the decay times $\tau_{\text {ISO }}$ and $\tau_{\mathrm{AGG}}$ were treated as global parameters, i.e. kept constant as a function of the wavelength, while the amplitudes

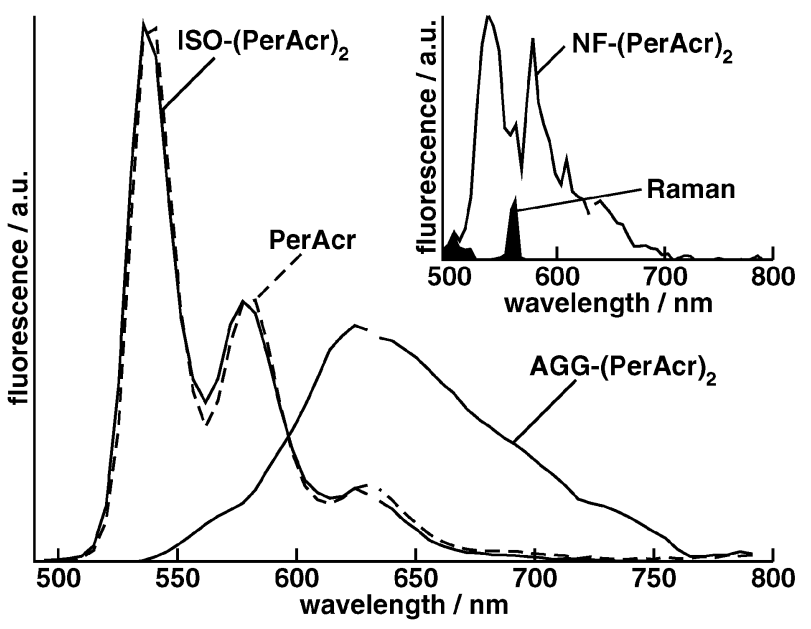

Fig. 6 Decay-associated spectra (DAS) from ISO-(PerAcr) $)_{2}$ and AGG-(PerAcr) $)_{2}$ (solid lines). For comparison, the DAS of the PerAcr emission is also shown (dashed line). The inset shows the DAS for the unresolved processes (solid line) as well as the Raman spectrum from a pure toluene solution obtained in a reference experiment (black, filled). The interruptions in the DAS around $630 \mathrm{~nm}$ shows where the two separately measured spectral ranges were joined. The DAS are normalised to the area. The Raman spectrum is scaled according to its contribution to the DAS for the unresolved processes.

were fitted separately for each wavelength interval providing the decay-associated spectra (DAS) for each decay time.

The resulting decay times are $\tau_{\mathrm{ISO}}=3.7 \mathrm{~ns}$ and $\tau_{\mathrm{AGG}}=27 \mathrm{~ns}$. The corresponding DAS of ISO-(PerAcr) $)_{2}$ and AGG-(PerAcr) are shown in Fig. 6, together with the DAS of PerAcr featuring only one decay component of $4.0 \mathrm{~ns}$. The DAS for ISO-(PerAcr) shows a remarkable resemblance with the DAS from PerAcr with bands peaking at $540 \mathrm{~nm}, 580 \mathrm{~nm}$, and $630 \mathrm{~nm}$, in close agreement with the steady-state PerAcr emission spectrum, see Fig. 2b. For AGG-(PerAcr $)_{2}$ the DAS shows a featureless band peaking at $630 \mathrm{~nm}$ with a width of $95 \mathrm{~nm}$ (FWHM). The results from this analysis support the above made assignments and allow unravelling the contributions from the individual spectral components to the emission spectrum. Considering the absolute amplitudes of the DAS, we find that the total emission of AGG-(PerAcr) 2 , i.e. integrated over wavelength and time, exceeds the total emission of ISO-(PerAcr) $)_{2}$ by about $10 \%$.

Additionally, the global analysis of the 186 decays enables us to obtain the DAS for all unresolved contributions, which has some similarity with the DAS from ISO-(PerAcr) $)_{2}$, as shown in the inset in Fig. 6. The black area superimposed in the inset corresponds to the Raman spectrum of the solvent and can account only for a very small fraction of this spectrum. At first sight the discovery of a further component contributing to the fluorescence seems to be at odds with the observation of isosbestic points in the fluorescence-excitation spectra and the conclusions about exactly two fluorescing species. However, it has to be kept in mind that fluorescenceexcitation spectroscopy is not sensitive to non-fluorescing absorbers. Since the DAS from the unresolved, fast component corresponds to a very weak signal due to the fast quenching (decay time below $30 \mathrm{ps}$ ) this component is not observed in a steady-state fluorescence-excitation spectrum. 


\section{Fluorescence-correlation spectroscopy}

Further information about the emitting species can be obtained from the correlation experiments. For all correlation curves we find a dip at lag time zero, a fast decay on the submicrosecond time scale, and a decay on the time scale of some ten microseconds. In accordance with literature data we ascribe the dip at zero lag time to the well-known photon antibunching, ${ }^{16,48,49}$ and the slow decay at longer times to the diffusion of the fluorescing particle out of the focal volume..$^{50-52}$ Yet the clear difference on the submicrosecond time scale between the autocorrelations recorded in the green and red spectral regions, Fig. 4a and b, provides further evidence for the presence of two emitting species. The non-vanishing crosscorrelation between the green and red channel tells us that these two emitting species exist on one individual molecule and can be inter-converted into each other. Together with the assignment of the two fluorescing species to the isolated and $\pi$-stacked PBI subunits made above, we conclude that the fast decay of the correlations on the submicrosecond time scale reflects the dynamics of the $\pi-\pi$ stacking process.

In order to quantify this statement, we performed a global analysis of the entire data set from the correlation experiments. We modelled the correlation functions $G^{(\mathrm{m}, \mathrm{n})}(\tau)(\mathrm{m}, \mathrm{n}=$ red,green) as the product of two components: $G_{\text {diff }}^{(\mathrm{m}, \mathrm{n})}(\tau)$, corresponding to the diffusion of single (PerAcr $)_{2}$ molecules through the confocal volume and $G_{\mathrm{mol}}^{(\mathrm{m}, \mathrm{n})}(\tau)$ corresponding to correlations that originate from the intrinsic molecular dynamics (antibunching and/or conformational dynamics), and convoluted $(\otimes)$ the result with the instrument response function $\operatorname{IRF}(\tau)$. Hence

$$
G^{(\mathrm{m}, \mathrm{n})}(\tau)=\left(G_{\mathrm{diff}}^{(\mathrm{m}, \mathrm{n})}(\tau) G_{\mathrm{mol}}^{(\mathrm{m}, \mathrm{n})}(\tau)\right) \otimes \operatorname{IRF}(\tau)
$$

In the following, only $\tau \geq 0$ will be discussed, because the autocorrelations can be assumed to be symmetric, and the crosscorrelation can be rewritten as

$$
G^{(\text {red,green })}(\tau)=\left\{\begin{array}{ll}
G^{(\text {red,green })}(\tau), & \tau \geq 0 \\
G^{\text {(green,red })}(-\tau), & \tau<0
\end{array} .\right.
$$

The IRF is represented by a triangular function with a full width at a half maximum of $10 \mathrm{~ns}$ and the diffusion part is treated as ${ }^{50-52}$

$$
G_{\mathrm{diff}}^{(\mathrm{m}, \mathrm{n})}(\tau)=D^{(\mathrm{m}, \mathrm{n})}\left(1+\frac{\tau}{\tau_{D}^{(\mathrm{m}, \mathrm{n})}}\right)^{-1}
$$

where $D^{(\mathrm{m}, \mathrm{n})}$ is the contrast and $\tau_{D}^{(\mathrm{m}, \mathrm{n})}$ the diffusion time that was set to the experimentally obtained half time of the long decay of the respective correlation curve. This contribution is assumed to be symmetric for the crosscorrelation function, i.e. $D^{\text {(red,green) }}=D^{\text {(green,red) }}, \tau_{D}^{\text {(red,green) }}=\tau_{D}^{\text {(green,red) }}$.

The intensity correlation functions are defined as

$$
G_{\mathrm{mol}}^{(\mathrm{m}, \mathrm{n})}(\tau)=\frac{\left\langle I^{(\mathrm{m})}(t) I^{(\mathrm{n})}(t+\tau)\right\rangle}{I^{(\mathrm{m})} I^{(\mathrm{n})}}
$$

Here and in the following, the angular brackets symbolize the ensemble average and the time-independent terms denote the steady-state value, respectively.

In order to find expressions for $G_{\mathrm{mol}}^{(\mathrm{m}, \mathrm{n})}(\tau)$ we have to take into account that we deal with two fluorescing species: ISO-(PerAcr) $)_{2}$ which emits preferentially into the green detection channel and AGG-(PerAcr $)_{2}$ which emits preferentially into the red detection channel. However, due to cross talk an unambiguous assignment between the detection channels and the molecular conformations becomes impossible. Consider a molecule that can reside in different conformations numbered with the index $\mathrm{K}$ (here: ISO and AGG) and a detection system that distinguishes between several spectrally resolved channels numbered with the index $\mathrm{m}$ (here: green and red). Then the total fluorescence intensity detected in channel $\mathrm{m}, I^{(\mathrm{m})}(t)$, can be written as

$$
I^{(\mathrm{m})}(t)=\sum_{\mathrm{K}} p_{\mathrm{K}}(t) \xi_{\mathrm{K}}^{(\mathrm{m})}(t)
$$

where $p_{\mathrm{K}}(t)=1$, or 0 depending on whether the emitting molecule resides in conformation $\mathrm{K}$ or not, $\xi_{\mathrm{K}}^{(\mathrm{m})}(t)$ refers to the intensity emitted into detection channel $\mathrm{m}$ while the molecule resides in conformation $\mathrm{K}$, and the sum runs over all conformations. Substituting eqn (6) in eqn (5), we find

$$
\begin{aligned}
G_{\mathrm{mol}}^{(\mathrm{m}, \mathrm{n})}(\tau) & =\sum_{\mathrm{K}, \mathrm{L}} \frac{\left\langle\xi_{\mathrm{K}}^{(\mathrm{m})}(t) p_{\mathrm{K}}(t) \xi_{\mathrm{L}}^{(\mathrm{n})}(t+\tau) p_{\mathrm{L}}(t+\tau)\right\rangle}{I^{(\mathrm{m})} I^{(\mathrm{n})}} \\
& =\sum_{\mathrm{K}, \mathrm{L}} \frac{\left\langle\xi_{\mathrm{K}}^{(\mathrm{m})}(t) \xi_{\mathrm{L}}^{(\mathrm{n})}(t+\tau)\right\rangle\left\langle p_{\mathrm{K}}(t) p_{\mathrm{L}}(t+\tau)\right\rangle}{I^{(\mathrm{m})} I^{(\mathrm{n})}}
\end{aligned}
$$

Defining the signal correlation function

$$
S_{\mathrm{KL}}(\tau)=\frac{\left\langle\xi_{\mathrm{K}}^{(\mathrm{m})}(t) \xi_{\mathrm{L}}^{(\mathrm{n})}(t+\tau)\right\rangle}{\xi_{\mathrm{K}}^{(\mathrm{m})} \xi_{\mathrm{L}}^{(\mathrm{n})}}
$$

and the conformation correlation function

$$
P_{\mathrm{KL}}(\tau)=\frac{\left\langle p_{\mathrm{K}}(t) p_{\mathrm{L}}(t+\tau)\right\rangle}{p_{\mathrm{K}} p_{\mathrm{L}}}
$$

allows us to rewrite eqn (7) as

$$
\begin{aligned}
G_{\mathrm{mol}}^{(\mathrm{m}, \mathrm{n})}(\tau) & =\sum_{\mathrm{K}, \mathrm{L}} \frac{\xi_{\mathrm{K}}^{(\mathrm{m})} p_{\mathrm{K}}}{I^{(\mathrm{m})}} \frac{\xi_{\mathrm{L}}^{(\mathrm{n})} p_{\mathrm{L}}}{I^{(\mathrm{n})}} S_{\mathrm{KL}}(\tau) P_{\mathrm{KL}}(\tau) \\
& =\sum_{\mathrm{K}, \mathrm{L}} i_{\mathrm{K}}^{(\mathrm{m})} i_{\mathrm{L}}^{(\mathrm{n})} S_{\mathrm{KL}}(\tau) P_{\mathrm{KL}}(\tau)
\end{aligned}
$$

where $i_{\mathrm{K}}^{(\mathrm{m})}=\frac{\xi_{\mathrm{K}}^{(\mathrm{m})} p_{\mathrm{K}}}{I^{(\mathrm{m})}}$ denotes the relative contribution of conformation $\mathrm{K}$ to detection channel $\mathrm{m}$, and $\sum_{\mathrm{K}} i_{\mathrm{K}}^{(\mathrm{m})}=1$. In order to find an explicit expression for $G_{\mathrm{mol}}^{(\mathrm{m}, \mathrm{n})}(\tau)$ we have to specify the relative intensities $i_{\mathrm{K}}^{(\mathrm{m})}$, the signal correlation $S_{\mathrm{KL}}(\tau)$, and the population correlation $P_{\mathrm{KL}}(\tau)$, in more detail. As is obvious from the DAS, see Fig. 6, we have to expect some emission from ISO-(PerAcr $)_{2}$ into the red detection channel whereas crosstalk from AGG-(PerAcr $)_{2}$ into the green detection channel can be neglected. This yields the following correspondences: $i_{\mathrm{AGG}}^{(\mathrm{red})}=1-i_{\mathrm{ISO}}^{\text {(red) }}, i_{\mathrm{ISO}}^{\text {(green) }}=1, i_{\mathrm{AGG}}^{(\text {green })}=0$.

The signal correlation function $S_{\mathrm{KL}}(\tau)$ reflects the fluorescence antibunching and can be modelled as ${ }^{53}$

$$
S_{\mathrm{KL}}(\tau)=1-\mathrm{e}^{-\frac{\tau}{\tau_{\mathrm{L}}}}
$$

where $\tau_{\mathrm{L}}$ is the decay time of the emitting state in conformation L. In the following these were set to the experimentally obtained decay times $\tau_{\mathrm{ISO}}=3.7 \mathrm{~ns}$ and $\tau_{\mathrm{AGG}}=27 \mathrm{~ns}$. 
In order to obtain the population correlation functions $P_{\mathrm{KL}}(\tau)$, we tested different models for the conformational dynamics (see below) that were based on a system of linear differential equations of the type

$$
\frac{\mathrm{d}}{\mathrm{dt}}\left\langle p_{\mathrm{K}}(t)\right\rangle=-\left\langle p_{\mathrm{K}}(t)\right\rangle \sum_{\mathrm{L} \neq \mathrm{K}} k_{\mathrm{KL}}+\sum_{\mathrm{L} \neq \mathrm{K}}\left(k_{\mathrm{LK}}\left\langle p_{\mathrm{L}}(t)\right\rangle\right)
$$

where $\left\langle p_{\mathrm{K}}(t)\right\rangle$ and $\left\langle p_{\mathrm{L}}(t)\right\rangle$ represent the ensemble averages of the populations of the conformations that can be converted into each other with rates $k$. Since by definition $0 \leq\left\langle p_{\mathrm{K}}(t)\right\rangle \leq 1$, $\left\langle p_{\mathrm{K}}(t)\right\rangle$ is equivalent to the probability to find the molecule in conformation $\mathrm{K}$ at time $t$. Therefore, eqn (12) can be solved for the initial condition $\left\langle p_{\mathrm{K}}(t)\right\rangle=1$ and $\left\langle p_{\mathrm{L}}(t)\right\rangle=0$ for all $\mathrm{L} \neq \mathrm{K}$, providing the conditional probability $\left\langle p_{\mathrm{L}}(t+\tau) \mid p_{\mathrm{K}}(t)\right\rangle$ that the molecule is in conformation $\mathrm{L}$ at time $(t+\tau)$ if it was in conformation $\mathrm{K}$ state at time $t$. Yet, by definition of the conditional probability we have

$$
\left\langle p_{\mathrm{L}}(t+\tau) \mid p_{\mathrm{K}}(t)\right\rangle=\frac{\left\langle p_{\mathrm{L}}(t+\tau) p_{\mathrm{K}}(t)\right\rangle}{p_{\mathrm{K}}}
$$

and resubstitution into eqn (9) finally yields

$$
P_{\mathrm{KL}}(\tau)=\frac{\left\langle p_{\mathrm{L}}(t+\tau) \mid p_{\mathrm{K}}(t)\right\rangle}{p_{\mathrm{L}}}
$$

The first model that we consider for the conformational dynamics describes the interconversion of the conformations ISO-(PerAcr $)_{2}$ and AGG-(PerAcr $)_{2}$ into each other with rates $k_{\mathrm{IA}}$ and $k_{\mathrm{AI}}$, respectively, and corresponds to $\mathrm{K}, \mathrm{L}=\mathrm{ISO}$, AGG. This model will be referred to as I-A in the following, see Fig. 7a, and yields population-correlation functions of the type

$$
P_{\mathrm{KL}}(\tau)=1+\alpha_{\mathrm{KL}} \mathrm{e}^{-\lambda \tau}
$$

where $\lambda$ is the non-trivial eigenvalue of the system of linear differential eqn (11), and both $\alpha_{\mathrm{KL}}$ and $\lambda$ are functions of the transition rates $k_{\mathrm{IA}}$ and $k_{\mathrm{AI}}: \alpha_{\mathrm{ISO}, \mathrm{ISO}}=\frac{k_{\mathrm{IA}}}{k_{\mathrm{AI}}}, \alpha_{\mathrm{AGG}, \mathrm{AGG}}=\frac{k_{\mathrm{AI}}}{k_{\mathrm{IA}}}$, $\alpha_{\mathrm{ISO}, \mathrm{AGG}}=\alpha_{\mathrm{AGG}, \mathrm{ISO}}=-1$, and $\lambda=k_{\mathrm{IA}}+k_{\mathrm{AI}}$. Inserting eqn (4), (10), (11) and (15) into eqn (2), we performed a global analysis of the entire data set of all three correlation functions, treating the rates $k_{\mathrm{IA}}$ and $k_{\mathrm{AI}}$ as well as the crosstalk parameter $i_{\text {ISO }}^{\text {(red) }}$ as global parameters. The analysis was restricted to the time range $\pm 2.5 \mu \mathrm{s}$ around lag time zero. The best agreement between the model and the data was obtained for $k_{\mathrm{IA}}=1.6 \times 10^{7} \mathrm{~s}^{-1}, k_{\mathrm{AI}}=5 \times 10^{6} \mathrm{~s}^{-1}$ and $i_{\mathrm{ISO}}^{\text {(red) }}=0.08$; (a) I-A

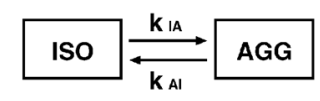

(b)
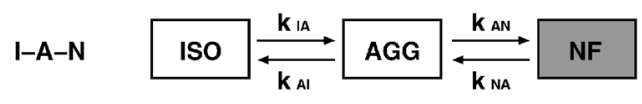

(c)

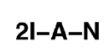

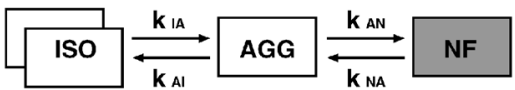

Fig. 7 Schematic of the models tested to describe the correlation data. The boxes refer to states that are interpreted as isolated PBI units (ISO), aggregated PBI units (AGG), and non-fluorescing PBI units (NF). These states are connected by transition rates as given in the figure. For the $2 \mathrm{I}-\mathrm{A}-\mathrm{N}$ model the state ISO consists of two independently emitting PBI units. the corresponding fit functions are shown in Fig. 4 by the thin lines. Obviously, this model fails to explain the observations between $50 \mathrm{~ns}$ and $500 \mathrm{~ns}$ (positive as well as negative lag time). Yet it explains the asymmetry of the crosscorrelation function around lag time zero, which obviously can be attributed to reflect solely photon antibunching.

The findings from time-resolved spectroscopy, where we observed additionally a heavily quenched, barely fluorescing state, led us to expand the model by a non-fluorescent state $\mathrm{NF}$-(PerAcr $)_{2}$. Generally this state might be connected with both or one of the conformations ISO-(PerAcr $)_{2}$ and AGG-(PerAcr $)_{2}$. We found best agreement with the experimental data for a model where we connect AGG-(PerAcr) $)_{2}$ to $\mathrm{NF}-(\mathrm{PerAcr})_{2}$ (rates $k_{\mathrm{AN}}$ and $\left.k_{\mathrm{NA}}\right)$ referred to as $\mathrm{I}-\mathrm{A}-\mathrm{N}$, Fig. 7b. This model yields correlation functions of the type

$$
P_{\mathrm{KL}}(\tau)=1+\beta_{\mathrm{KL}} \mathrm{e}^{-\lambda_{1} \tau}+\gamma_{\mathrm{KL}} \mathrm{e}^{-\lambda_{2} \tau}
$$

where $\lambda_{1}$ and $\lambda_{2}$ are the non-trivial eigenvalues of the linear differential eqn (11), and $\beta_{\mathrm{KL}}, \gamma_{\mathrm{KL}}, \lambda_{1}$ and $\lambda_{2}$ are functions of the transition rates $k_{\mathrm{IA}}, k_{\mathrm{AI}}, k_{\mathrm{AN}}$ and $k_{\mathrm{NA}}$. The analytical expressions have been obtained by use of the computer algebra system Maple (version 13), yet are omitted here for brevity. A similar global analysis as detailed above yielded $k_{\mathrm{IA}}=1.5 \times$ $10^{7} \mathrm{~s}^{-1}, k_{\mathrm{AI}}=7 \times 10^{6} \mathrm{~s}^{-1}, k_{\mathrm{AN}}=1 \times 10^{6} \mathrm{~s}^{-1}, k_{\mathrm{NA}}=4 \times 10^{6} \mathrm{~s}^{-1}$ and $i_{\mathrm{ISO}}^{\text {(red) }}=0.08$. The resulting functions (not shown) are in very good agreement with the experimental data-points with only one exception: the data-point at lag time zero for the autocorrelation of the emission in the green detection channel. This is shown in the inset of Fig. 4a on an expanded scale and it becomes clear that the photon antibunching contribution, i.e. the depth of the dip at lag time zero, is overestimated by this model.

Modelling of the photon antibunching was introduced based on the assumption that each (PerAcr) $)_{2}$ molecule carries exactly one emitter at a time. However, for the non-stacked conformation ISO-(PerAcr $)_{2}$ it is likely that we deal in fact with two isolated PBI units which can emit photons independently from each other, diminishing the photon antibunching dip. This can be taken into account by replacing $S_{\text {ISO,ISO }}(\tau)$ in eqn (11) by

$$
S_{\text {ISO }, \text { ISO }}(\tau)=1-\frac{1}{2} \mathrm{e}^{-\frac{\tau}{\tau_{\text {ISO }}}}
$$

which finally results in a model referred to as $2 \mathrm{I}-\mathrm{A}-\mathrm{N}$, Fig. $7 \mathrm{c}$. The best fit is obtained for $k_{\mathrm{IA}}=1.3 \times 10^{7} \mathrm{~s}^{-1}, k_{\mathrm{AI}}=8 \times 10^{6} \mathrm{~s}^{-1}$, $k_{\mathrm{AN}}=2 \times 10^{6} \mathrm{~s}^{-1}, k_{\mathrm{NA}}=4 \times 10^{6} \mathrm{~s}^{-1}$, and $i_{\text {ISO }}^{\text {(red) }}=0.09$. These fit functions are in very good agreement with the experimental data, as shown in Fig. 4 by the bold line. But as can be seen in the inset of Fig. 4a, while the I-A-N model overestimates the photon antibunching, this effect is slightly underestimated by the $2 \mathrm{I}-\mathrm{A}-\mathrm{N}$ model.

For clarity the contributions to the correlation function for the various models as well as the input parameters used are summarized in Table 1.

\section{Discussion}

From the transition rates that follow from the $2 \mathrm{I}-\mathrm{A}-\mathrm{N}$ model the relative steady-state populations of the states ISO-(PerAcr $)_{2}$, 
Table 1 Equations and parameters used for the models employed to analyse the fluorescence (cross) correlation curves shown in Fig. 4. \# refers to the index of the equations in the running text

\begin{tabular}{|c|c|c|c|c|}
\hline & Equation & $\#$ & Model & Parameters \\
\hline Correlation function & $\begin{array}{l}G^{(\mathrm{m}, \mathrm{n})}(\tau)=\left(G_{\mathrm{diff}}^{(\mathrm{m}, \mathrm{n})}(\tau) G_{\mathrm{mol}}^{(\mathrm{m}, \mathrm{n})}(\tau)\right) \\
\otimes \operatorname{IRF}(\tau)\end{array}$ & (2) & $\mathrm{I}-\mathrm{A}, \mathrm{I}-\mathrm{A}-\mathrm{N}, 2 \mathrm{I}-\mathrm{A}-\mathrm{N}$ & $\begin{array}{l}\mathrm{m}, \mathrm{n}=\text { green,red; } \operatorname{IRF}(\tau) \text { : triangular function, } \\
\text { FWHM } 10 \mathrm{~ns}\end{array}$ \\
\hline Diffusion & $G_{\mathrm{diff}}^{(\mathrm{m}, \mathrm{n})}(\tau)=D^{(\mathrm{m}, \mathrm{n})}\left(1+\frac{\tau}{\tau_{D}^{(\mathrm{m}, \mathrm{n})}}\right)^{-1}$ & (4) & $\mathrm{I}-\mathrm{A}, \mathrm{I}-\mathrm{A}-\mathrm{N}, 2 \mathrm{I}-\mathrm{A}-\mathrm{N}$ & $\begin{array}{l}D^{(\text {red,green })}=D^{(\text {green,red })} ; \tau_{D}^{\text {(green,green })}=60 \mu \mathrm{s}, \\
\tau_{D}^{(\text {red,red })}=60 \mu \mathrm{s}, \tau_{D}^{\text {(red,green) }}=\tau_{D}^{\text {(green,red })}=50 \mu \mathrm{s}\end{array}$ \\
\hline \multirow[t]{2}{*}{$\begin{array}{l}\text { Intrinsic molecular } \\
\text { dynamics }\end{array}$} & \multirow[t]{2}{*}{$G_{\mathrm{mol}}^{(\mathrm{m}, \mathrm{n})}(\tau)=\sum_{\mathrm{K}, \mathrm{L}} i_{\mathrm{K}}^{(\mathrm{m})} i_{\mathrm{L}}^{(\mathrm{n})} S_{\mathrm{KL}}(\tau) P_{\mathrm{KL}}(\tau)$} & \multirow[t]{2}{*}{$(10)$} & $\mathrm{I}-\mathrm{A}$ & $\begin{array}{l}\mathrm{K}, \mathrm{L}=\mathrm{ISO}, \mathrm{AGG} ; i_{\mathrm{AGG}}^{\mathrm{red}}=1-i_{\mathrm{ISO}}^{(\mathrm{red})}, i_{\mathrm{ISO}}^{(\text {green })}=1, \\
i_{\mathrm{AGG}}^{\text {(reen) }}=0\end{array}$ \\
\hline & & & $\mathrm{I}-\mathrm{A}-\mathrm{N}, 2 \mathrm{I}-\mathrm{A}-\mathrm{N}$ & $\begin{array}{l}\mathrm{K}, \mathrm{L}=\mathrm{ISO}, \mathrm{AGG}, \mathrm{NF} ; i_{\mathrm{AGG}}^{(\mathrm{red})}=1-i_{\mathrm{SSO}}^{\text {(red })} \\
i_{\mathrm{ISO}}^{\text {(green })}=1, i_{\mathrm{AGG}}^{(\text {green })}=0, i_{\mathrm{NF}}^{\text {(green })}=0, i_{\mathrm{NF}}^{\text {(red })}=0\end{array}$ \\
\hline \multirow{2}{*}{$\begin{array}{l}\text { Signal correlation } \\
\text { function (fluorescence } \\
\text { antibunching) }\end{array}$} & $S_{\mathrm{KL}}(\tau)=1-\mathrm{e}^{-\frac{\tau}{\tau_{\mathrm{L}}}}$ & (11) & $\begin{array}{l}\mathrm{I}-\mathrm{A}, \mathrm{I}-\mathrm{A}-\mathrm{N}, 2 \mathrm{I}-\mathrm{A}-\mathrm{N} \\
\left.\text { (except } S_{\text {ISO,ISO }}\right)\end{array}$ & $\tau_{\mathrm{ISO}}=3.7 \mathrm{~ns}, \tau_{\mathrm{AGG}}=27 \mathrm{~ns}$ \\
\hline & $S_{\mathrm{ISO}, \mathrm{ISO}}(\tau)=1-\frac{1}{2} \mathrm{e}^{-\frac{\tau}{\tau \mathrm{ISO}}}$ & (17) & $2 \mathrm{I}-\mathrm{A}-\mathrm{N}$ & $\tau_{\mathrm{ISO}}=3.7 \mathrm{~ns}$ \\
\hline \multirow[t]{2}{*}{$\begin{array}{l}\text { Conformation } \\
\text { correlation function }\end{array}$} & $P_{\mathrm{KL}}(\tau)=1+\alpha_{\mathrm{KL}} \mathrm{e}^{-\lambda \tau}$ & $(15)$ & $\mathrm{I}-\mathrm{A}$ & $\alpha_{\mathrm{ISO}, \mathrm{ISO}}=\frac{k_{\mathrm{IA}}}{k_{\mathrm{AI}}}, \alpha_{\mathrm{AGG}, \mathrm{AGG}}=\frac{k_{\mathrm{AI}}}{k_{\mathrm{IA}}}$ \\
\hline & $P_{\mathrm{KL}}(\tau)=1+\beta_{\mathrm{KL}} \mathrm{e}^{-\lambda_{1} \tau}+\gamma_{\mathrm{KL}} \mathrm{e}^{-\lambda_{2} \tau}$ & (16) & $\mathrm{I}-\mathrm{A}-\mathrm{N}, 2 \mathrm{I}-\mathrm{A}-\mathrm{N}$ & $\begin{array}{l}\alpha_{\mathrm{ISO}, \mathrm{AGG}}=\alpha_{\mathrm{AGG}, \mathrm{ISO}}=-1, \lambda=k_{\mathrm{IA}}+k_{\mathrm{AI}} \\
\text { Analytical expressions for } \beta_{\mathrm{KL}}, \gamma_{\mathrm{KL}}, \lambda_{1} \\
\text { and } \lambda_{2} \text { not shown; obtained by use of the } \\
\text { computer algebra system Maple (version } 13 \text { ) }\end{array}$ \\
\hline
\end{tabular}

AGG-(PerAcr) $)_{2}$, and NF-(PerAcr $)_{2}$ can be deduced to be $29 \%, 50 \%$, and $21 \%$, respectively. The relative population of $29 \%$ for ISO-(PerAcr $)_{2}$ is in very good agreement with the decrease of the intensity of the PerAcr-like component in the (PerAcr $)_{2}$ emission spectrum by a factor of three with respect to the intensity of the emission from a pure PerAcr sample. Association of the species $\mathrm{NF}-(\mathrm{PerAcr})_{2}$ with the heavily quenched, barely fluorescent state observed in the time-resolved experiments, and the tentative assumption that the absorption spectrum of $\mathrm{NF}$-(PerAcr $)_{2}$ resembles the absorption spectrum of ISO-(PerAcr $)_{2}$, explains straightforwardly the successful reconstruction of the (PerAcr $)_{2}$ absorption spectrum, see Fig. 5, by equal fractions from the aggregated and nonaggregated species. The assumption about the resemblance of the absorption spectra for NF-(PerAcr) $)_{2}$ and ISO-(PerAcr) $)_{2}$ is motivated by the fact that the DAS from the unresolved fast decays, see inset Fig. 6, shows clear similarities with the DAS from ISO-(PerAcr) $)_{2}$, which makes it likely that both species feature also very similar absorption spectra.

Although not a proof, the interpretation so far yields a consistent picture about the states involved, which finally brings us to the question about the character of the quenched/ non-fluorescing state. One option is that NF-(PerAcr) $)_{2}$ can be associated with a charge-separated state. Experiments of nonaggregated PBI dimers in polystyrene films revealed a photoinduced charge transfer from the matrix to the PBIs that was detected via the quenching of the fluorescence. ${ }^{54}$ However, in the nonpolar solvent toluene such a process is not very likely. Also, in absorption experiments up to $1100 \mathrm{~nm}$, we did not find any indication for a charge-transfer band hinting at the possibility of photo-induced charge transfer, or the absorption of a steadystate population of anionic PBI. Another possibility is that the state $\mathrm{NF}-(\mathrm{PerAcr})_{2}$ corresponds to a triplet state. For monomeric perylene derivatives it is well known that the triplet yield is negligible. ${ }^{55-57}$ However, for aggregated perylenes considerable triplet yields have been reported, ${ }^{40}$ i.e. a significant population of the triplet state cannot be excluded a priori. In fact, the triplet state being only accessible from the aggregated state would be consistent with the model derived from the correlation data. However, for $\mathrm{NF}$-(PerAcr $)_{2}$ being a triplet state one would expect a strong dependence of the correlation functions on the excitation intensity. Variation of this parameter within an order of magnitude did not have any influence on the correlations. Moreover, the dynamics we found in (PerAcr) $)_{2}$ occurs on timescales well below $1 \mu \mathrm{s}$, whereas the triplet lifetimes reported for PBI and aggregated PBI are much longer $(\sim 100 \mu \mathrm{s}){ }^{40,58}$ Finally, the spectral signatures, i.e. the PerAcr-like DAS as well as the assumed PerAcr-like absorption for the quenched state, are clearly not compatible with the interpretation of NF-(PerAcr) as a triplet state. In summary, we exclude the association of the non-fluorescing state with both a charge-separated and a triplet state. Based on the proposed conformation dynamics, Fig. 7c, it can be speculated that the non-fluorescent state is the "truly" aggregated state of (PerAcr) ${ }_{2}$, and the state that we termed AGG is only an intermediate state. However, on the basis of the available data, the nature of the non-fluorescent state remains an open question and deserves further attention in the future.

\section{Acknowledgements}

We gratefully acknowledge financial support from the German Science Foundation (DFG; GRK-1640).

\section{References}

1 G. F. Brown and J. Wu, Laser Photonics Rev., 2009, 3, 394.

2 A. A. Rockett, Curr. Opin. Solid State Mater. Sci., 2010, 14, 117.

3 J.-L. Brédas and J. R. Durrant, Acc. Chem. Res., 2009, 42, 1689.

4 C. G. Hübner, V. Ksenofontov, F. Nolde, K. Müllen and T. Basché, J. Chem. Phys., 2004, 120, 10867.

5 M. Cotlet, S. Masuo, G. Luo, J. Hofkens, M. van der Auweraer, J. Verhoeven, K. Müllen, X. S. Xie and F. de Schryver, Proc. Natl. Acad. Sci. U. S. A., 2004, 101, 14343. 
6 T. Körzdörfer, S. Tretiak and S. Kümmel, J. Chem. Phys., 2009, 131, 034310.

7 C. C. Hofmann, P. Bauer, S. A. Haque, M. Thelakkat and J. Köhler, J. Chem. Phys., 2009, 131, 144512.

8 C. C. Hofmann, S. M. Lindner, M. Ruppert, A. Hirsch, S. A. Haque, M. Thelakkat and J. Köhler, J. Phys. Chem. B, 2010, 114, 9148.

9 R. Métivier, F. Kulzer, T. Weil, K. Müllen and T. Basché, J. Am. Chem. Soc., 2004, 126, 14364.

10 F. C. de Schryver, T. Vosch, M. Cotlet, M. van der Auweraer, K. Müllen and J. Hofkens, Acc. Chem. Res., 2005, 38, 514.

11 I. G. Scheblykin, O. Y. Sliusarenko, L. S. Lepnev, A. G. Vitukhnovsky and M. van der Auweraer, J. Phys. Chem. B, 2000, 104, 10949.

12 C. Didraga, A. Pugzlys, P. R. Hania, H. von Berlepsch, K. Duppen and J. Knoester, J. Phys. Chem. B, 2004, 108, 14976.

13 E. Lang, A. Sorokin, M. Drechsler, Y. V. Malyukin and J. Köhler, Nano Lett., 2005, 5, 2635.

14 D. M. Eisele, J. Knoester, S. Kirstein, J. P. Rabe and D. A. Vanden Bout, Nat. Nanotechnol., 2009, 4, 658.

15 F. Würthner, T. E. Kaiser and C. R. Saha-Möller, Angew. Chem., Int. Ed., 2011, 50, 3376.

16 J. Hernando, J. P. Hoogenboom, E. M. H. P. van Dijk, J. J. GarciaLopez, M. Crego-Calama, D. N. Reinhoudt, N. F. van Hulst and M. F. Garcia-Parajo, Phys. Rev. Lett., 2004, 93, 6404.

17 A. Sautter, B. K. Kaletas, D. G. Schmid, R. Dobrawa, M. Zimine, G. Jung, I. H. M. van Stokkum, L. de Cola, R. M. Williams and F. Würthner, J. Am. Chem. Soc., 2005, 127, 6719.

18 F. Würthner, C. C. You and C. R. Saha-Möller, Chem. Soc. Rev., 2004, 33, 133

19 A. Satake and Y. Kobuke, Org. Biomol. Chem., 2007, 5, 1679.

20 T. S. Balaban, Acc. Chem. Res., 2005, 38, 612.

21 G. D. Scholes and G. Rumbles, Nat. Mater., 2006, 5, 683.

22 J. Roncali, Acc. Chem. Res., 2009, 42, 1719.

23 J.-L. Brédas, J. E. Norton, J. Cornil and V. Coropceanu, Acc. Chem. Res., 2009, 42, 1691.

24 F. Würthner, Pure Appl. Chem., 2006, 78, 2341.

25 H. Langhals, Helv. Chim. Acta, 2005, 88, 1309.

26 G. Horowitz, F. Kouki, P. Spearman, D. Fichou, C. Nogues, X. Pan and F. Garnier, Adv. Mater., 1996, 8, 242.

27 S. Hüttner, M. Sommer and M. Thelakkat, Appl. Phys. Lett., 2008, 92, 093302 .

28 T. Kircher and H. G. Löhmannsröben, Phys. Chem. Chem. Phys., 1999, 1, 3987.

29 C. W. Tang, Appl. Phys. Lett., 1985, 48, 183.

30 Y. Liu, K.-R. Wang, D.-S. Guo and B.-P. Jiang, Adv. Funct. Mater., 2009, 19, 2230.

31 Y.-B. Ruan, A.-F. Li, J.-S. Zhao, J.-S. Shen and Y.-B. Jiang, Chem. Commun., 2010, 46, 4938.

32 H. Wang, D. Wang, Q. Wang, X. Li and C. A. Schalley, Org. Biomol. Chem., 2010, 8, 1017.

33 X. Zhang, S. Rehm, M. M. Safont-Sempere and F. Würthner, Nat. Chem., 2009, 1, 623.
34 T. Cordes, J. Vogelsang, M. Anaya, C. Spagnuolo, A. Gietl, W. Summerer, A. Herrmann, K. Müllen and P. Tinnefeld, J. Am. Chem. Soc., 2010, 132, 2404.

35 S. Rocha, J. A. Hutchison, K. Peneva, A. Herrmann, K. Müllen, M. Skjøt, C. I. Jørgensen, A. Svendsen, F. C. de Schryver, J. Hofkens and H. Uji-i, ChemPhysChem, 2009, 10, 151.

36 R. Samudrala, X. Zhang, R. M. Wadkins and D. L. Mattern, Bioorg. Med. Chem., 2007, 15, 186.

37 F. Würthner, Chem. Commun., 2004, 1564.

38 C. Hippius, I. H. M. van Stokkum, E. Zangrando, R. M. Williams, M. Wykes, D. Beljonne and F. Würthner, J. Phys. Chem. C, 2008, 112, 14626.

39 C. C. Hofmann, S. M. Lindner, M. Ruppert, A. Hirsch, S. A. Haque, M. Thelakkat and J. Köhler, Phys. Chem. Chem. Phys., 2010, 12, 14485.

40 D. Veldman, S. M. A. Chopin, S. C. J. Meskers, M. M. Groeneveld, R. M. Williams and R. A. J. Janssen, J. Phys. Chem. A, 2008, 112, 5846.

41 J. M. Giaimo, J. V. Lockard, L. E. Sinks, A. M. Scott, T. M. Wilson and M. R. Wasielewski, J. Phys. Chem. A, 2008, 112, 2322.

42 S. M. Lindner, N. Kaufmann and M. Thelakkat, Org. Electron., $2007, \mathbf{8}, 69$.

43 I. A. Howard, F. Laquai, P. E. Keivanidis, R. H. Friend and N. C. Greenham, J. Phys. Chem. C, 2009, 113, 21225-21232.

44 M. Sommer, S. M. Lindner and M. Thelakkat, Adv. Funct. Mater., 2007, 17, 1493.

45 H. Brown and R. Q. Twiss, Nature, 1956, 177, 27.

46 Z. Chen, V. Stepanenko, V. Dehm, P. Prins, L. D. A. Siebbeles, J. Seibt, P. Marquetand, V. Engel and F. Würthner, Chem.-Eur. J., $2007,13,436$

47 A. Tolkki, E. Vuorimaa, V. Chukharev, H. Lemmetyinen, P. Ihalainen, J. Peltonen, V. Dehm and F. Würthner, Langmuir. 2010, 26(9), 6630 .

48 Th. Basché, W. E. Moerner, M. Orrit and H. Talon, Phys. Rev. Lett., 1992, 69, 1516.

49 C. Hettich, C. Schmitt, J. Zitzmann, S. Kühn, I. Gerhardt and V. Sandoghdar, Science, 2002, 298, 385.

50 E. L. Elson and D. Madge, Biopolymers, 1974, 13, 1.

51 E. Haustein and P. Schwille, Ann. Rev. Biophys. Biomol. Struct., 2007, 36, 151.

52 J. Widengren and Ü. Mets, in Single Molecule Detection in Solution, ed. Ch. Zander, J. Enderlein and R. A. Keller, WILEY-VCH, Berlin, 1st edn, 2002, ch. 3, pp. 69-120.

53 L. Fleury, J. M. Segura, G. Zumofen, B. Hecht and U. P. Wild, Phys. Rev. Lett., 2000, 84, 1148.

54 D. Ernst, R. Hildner, C. Hippius, F. Würthner and J. Köhler, Chem. Phys. Lett., 2009, 482, 93.

55 N. Nijegorodov and D. P. Winkoun, Spectrochim. Acta, Part A, 1997, 53, 2013.

56 E. Lang, F. Würthner and J. Köhler, ChemPhysChem, 2005, 6, 935.

57 E. Lang, R. Hildner, H. Engelke, P. Osswald, F. Würthner and J. Köhler, ChemPhysChem, 2007, 8, 1487.

58 W. E. Ford and P. V. Kamat, J. Phys. Chem., 1987, 91, 6373. 